\title{
Which new semantic for new shapes?
}

\author{
B. Colajanni, G. Pellitteri \& S. Concialdi \\ Dipartimento di Progetto e Costruzione Edilizia, \\ Università di Palermo, Italy
}

\begin{abstract}
There are two innovations which have drastically changed the building process: the operational continuity of the design and construction phases, and the software allowing not only the representation but also the autonomous creation of complex shapes never before thought of just because they could not be represented. This last innovation gave rise to a new design paradigm whose tools, according to their supporters, are the most advanced fields of mathematics and information science. Some ways of using these new possibilities gave rise to a radical, problematic, change in the relationship net between the designer's intentions, the shapes through which they express them (invented or self-generated) and their semantic contents. The most radical position skips the problem denying the necessity of such a semantic content. A further question is raised when the context is thought to intervene directly in the shaping process of a building envelope.

Translating cultural influences into physical entities directly acting in transforming surfaces shapes entails a strongly idiosyncratic interpretation. Constructing a semantic code of shapes and context forces common to the sender (the architect that decides the shape) and the receiver of the communication (the social community in which the architecture is immersed) requires from the former a careful reflection on the meanings also beyond the sender's intentions, with which the community reads the designed shapes, according to its cultural standard.

The complexity of those processes has for a long time been the object of much debate. Some statements about the new paradigm seem to be metaphors rather than realities. Our contribution tries to detect some misunderstandings which a displaced use of some concepts has created have this nature. A design experiment is presented, that has been used as a test.

Keywords: digital architecture, topology, morphing, context forces.
\end{abstract}




\section{Introduction}

There are two innovations which have deeply changed the building process: the operational continuity of the design and construction phases, and the software allowing not only the representation but also the autonomous creation of complex shapes never before thought of, just because they could not be represented. The former is by now a widespread, almost universal, part of any building processes. The latter has experienced a different reception. Some architects smoothly introduced the new software into their design habits, drawing out of it all the instrumental capabilities offered. Some others, conjugating these possibilities with their eager interest for contemporary science and philosophy, have claimed the birth of a new architectural paradigm or, better, "the" new architecture paradigm, the only one allowing, at present, to "think architecture" an expression of Gilles Deleuze the present philosophical compass of the new architects. The well-known assertion of Gregg Lynn: "The nineties started angular and ended curvilinear. In Architecture started Decostructivist and ended topological" [1] summarizes this position well. Such a "fundamentalist" paradigm would deny architects like Renzo Piano or Tadao Ando the right of representing a valid alternative approach to design. Of course this claim of exclusivity can hardly be shared. However some arguments supporting the claim have deep theoretical implications, and deserve consideration. Others are mere metaphors, and are to be interpreted as such. Although widely discussed, a short summary of these metaphors can be useful in order to get rid of the misunderstanding they convey. This is done in Section 2. In Section 3 the main tool of the new paradigm "deformation" is examined. Section 4 signals the crucial problem of meaning. Section 5 refers to an experiment aimed at verifying some theses concerning the relationship shape-context forces.

\section{Magic words and metaphors}

Mathematics, and mainly Geometry has had, throughout history, constant relationships with architecture. Fruitful relationships, as long as its theories were directly translated into real, physical applications. The present interest seems to have a somewhat different character. Abstract, conventional mathematical concepts, are attributed as properties to architectural spaces or design procedures; and the fitness of the attribution is often questionable.

The enthusiasm for mathematics and philosophy is such as to graze infatuation. Mathematics is the kernel of the actual graphic software. The current use of this software has perhaps been felt by some architects as a frequentation of advanced mathematics. From this feeling the architects may have derived the sensation of having entered, as protagonists, a cultural environment of which mathematics is the core (together with philosophy). The relationship looks more metaphorical than real. Metaphors are free from the obligation of a deep understanding of the concepts, as well as of their real application. They allow an easy appropriation of the "aura" of up-to-date science and the following positive connotation; that is just what the architects pretend. 
A dictionary of the seminal words of the new architecture is certainly comprised of the following terms: non-Euclidean geometry, topology, dynamics, morphing unpredictability. Their use, as formerly said, is not exactly corresponding to their current meaning. A short analysis of a few of them is carried out in order to better grasp the key for understanding this kind of dictionary.

Non-Euclidean Geometry is an expression to which more than one meaning is attached. An easy remark could be that the geometry of a sphere is elliptical, hence, non Euclidean. It would be daring to infer that any spherical vault is a piece of "new architecture". The same can be said of all the analytical surfaces, such as quadrics, torus, and so on. On any surface, except on the plan, the possible geometry is non Euclidean. The "new architects" privilege a typology of surfaces (the NURBS) on which the non-validity of the Euclidean Geometry is pure triviality. Analogue misuse seems to be made of the word Cartesian: the formal aspect of orthogonal-based architectures is taken as consequences of the orthogonality of the Cartesian coordinated axes. The combination of the anathema to Euclidean geometry and to Cartesian space generates the following "disequation": [Euclidean Architecture + Cartesian Reference System] $\rightarrow$ modernist, rectilinear-orthogonal architecture $\rightarrow$ old architecture $\rightarrow$ unable to represent contemporary culture $<<$ [non-Euclidean, curvilinear, topological, morpho-generated Architecture] $\rightarrow$ representative of the contemporary cultures $\rightarrow$ in touch with the actual science and philosophy.

Another interpretation of non-Euclidean geometry describes it as a fourdimensional (or greater) geometry. Again, it is easy to observe that Computer Graphics allows one to draw only two-dimensional projections of geometrical four-dimensional (or greater) geometric entities which will always remain nothing more than projections on a two-dimensional space. Three-dimensional projections, when possible, as, for instance, is the case of the hypercube, are but assemblages of 3D volumes. Why then to refer to four-dimensional spaces that cannot become real architecture?

Sometimes, time is the fourth dimension referred to. Again, an unclear interpretation may be a synonym of dynamics or else the concept chain: time $\rightarrow$ change $\rightarrow$ dynamics $\rightarrow$ topology $\rightarrow$ morphing $\rightarrow$ new architecture. However, this is not a novelty. Both Guillaume Apollinaire and Marcel Duchamp since 1912-1913 mention the fourth dimension of the non-Euclidean Geometry as the matrix of their space [2]. The same was done by the Italian futurists.

Regard Topology a simple definition describes it as "the mathematical study of the properties that are preserved through deformations, twisting and stretching of objects. Tearing however is not allowed" [3]. Deformation is the key word which leads to the architectural use of topology. From topological point of view a sphere, a cube as well as all convex polyhedra are topologically equivalent since all of them have 2 as Euler characteristic value. No "new architect" would consider these surfaces as architectonically equivalent or, worse, equivalent to a deformed surface of an envelope designed by him. What "topological architects" care for is software that is able to deform shapes. The aim is to obtain surfaces not otherwise obtainable or thinkable if not already seen 
after the unpredictable generation and representation. The task is the deformed surface, the final result, not the procedure. At the end of the process, whatever is the generating procedure there is a NURBS surface, unavoidably existing in the scorned Euclidean space and described by means of the Cartesian coordinates of the control points. In the new architecture jargon, the emphasis is on the concept of transformation. In other words, the shapes must not be common, simple, or recognizable. They must be transformed, deformed, and unequivocally recognized as such. It is like transformation and deformation guarantee the necessary transgression of the "dull" order attributed to "Euclidean geometry" Counter-check. Gehry's surfaces are created with somewhat handicraft methods, not deforming but directly forming the architectural models. They may look, to eyes wanting to see that, as deformed, but they are not. Forms are similar, the creation process is not. Then, if the positive connotation derives from the process of deformation, it cannot be attributed to these forms. However, they are absolutely akin to the ones obtained by deformation. Conclusive is the witness of Jim Glimph of Gehry's studio on the first design of the Los Angeles Walt Disney Auditorium: "Study models were generated very quickly, spontaneously, around the basic ideas of the project. The development of sail-like forms and that kind of imagery was all done in physical model. There was no computer modeling at all. In fact, at that time, there were no computers in the office" [4]. Hence the nature of those forms did not depend on the use of computers. And hence again, the assertion that these forms are topological is that they have a non-Euclidean geometrical-topological ontology is not at all demonstrated. Just the contrary of what, M. Emmer asserts, i.e. that Gehry's Guggenheim Museum in Manhattan is "an even more topological project than that of the new Guggenheim museum in Bilbao" [5]. William Mitchell views Gehry's iterative multimedia process as "far more revolutionary" [6]. The thesis is contested by Lenoir and Alt: "he is in fact doing nothing revolutionary" [7].

Morphing is but the set of procedure of obtaining deformed shapes.

A summary of the theoretical bases of "new architecture" may be found in the following Kolarevic' sentence [8]: "The defining element of the topological architecture is its departure from the Euclidean geometry of discrete volumes represented in Cartesian space, and the extensive use of topological (rubbersheet geometry) of continuous curves and surfaces, mathematically described as NURBS'.

\section{Deformation as a design criterion}

There are two different ways of deforming shape. The grounding character of the first is the control of the start and the end shape of the process. Moreover, there are yet two variants of the procedure. One of them is the classical morphing. You start from a known shape, you choose an arrival shape, and the procedure builds as many intermediate shapes as you want. In some way you can foresee the type of shapes you will get. The result depends on the difference between starting and final shape. If the latter is a transformation of the former, the purpose is choosing the fittest hue in a field of substantially homogeneous solutions. If starting and 
final shapes are thoroughly different the aim is a true exploration of possibly unexpected results that highly divergent formal concepts may generate out of their reciprocal reaction.

The second way also has two variants. The starting point is for both a tentative shape. One variant commits the deformation to purely conventional, geometrical procedural rules; the second commits the deformation to a system of forces directly acting on the shape. The simulation is carried on a model endowed with virtual, global mechanical properties. Virtual mechanical forces (also possible metaphors of non-physical influences) are expected to produce virtual mechanical deformations. The aim is ambitious, the simulation of the effect that the context exerts on the shape. Some perplexities raise naturally. Let us refer to Franken's words: “Admittedly, we cannot grasp forces directly with our senses, but can only infer them through their effects. Our experience, however made is very sensitive to deformations that correspond to a natural play of forces. Our perception is thus conditioned toward forces, and uses them to interpret shapes. Deformed forms carry information about the forces at their origin" [8]. Substantially, Franken affirms the "a priori" impossibility of grasping the system of context forces before their action on the objects. From their effects they can only be inferred, not known. Moreover, information about the origin of the forces can be drawn only interpreting the deformation with our sensibility. So, if the hearth of the design procedure is the deformation effect of such a force system it is difficult to avoid the rise of some perplexities. How to foresee the system of forces to which the designed shape has to be submitted, if also its origin can be inferred only from occurred deformation? And, overall, how to translate non-physical forces into mechanical (physical) models of action and reaction? Isn't the determination of the force systems, supposedly representing the influence of the context, somewhat hydiosincratic, largely subjective, and, eventually, arbitrary inasmuch as it is not susceptible of popperian validation? And how to decide the degree of virtual deformability of the shape? Also an interactive procedure generating a series of refined solution cannot escape the arbitrariness of guessing the system of context forces. Notwithstanding all those uncertainties Franken has no doubts: "The forms we generate are never arbitrary, they can be explained and are subject to rationalisation" [9]. We maintain our perplexities on the real correspondence of the obtained results to the asserted procedure independently of their architectural value, which is, in the end, the only thing that has importance.

\section{The meaning}

Another question is the meaning these architectures can express. A very complex question, that of course we have neither intention nor claim to discuss. Nonetheless, we will express some remarks.

The question of meaning is linked with more than one concept of the new architects' theorisations: unpredictability, the intentional seek of indeterminacy, direct action of environment forces in the design process. A form, however generated, is perceived. Perception involves vision. Which is, using the words of 
Eisenman: "linking seeing to thinking, the eye to the mind" [10]. A malicious reader could recognize in this assertion an echo of the wölflinian purovisibilism [11]; from Kolarevic's "Back to the future" to an Eisenman's "Forward to the past"? In the same text he proposes to "detach what one sees from what one knows, the eye from the mind". It seems really doubtful to consider this proposed first step as possible. The purpose of this detachment is to skip the difficulties that the vision of a unpredicted and hence at least partially uncontrolled shape may meet "When the environment is inscribed or folded in such a way, the individual no longer remains the discursive function, the individual is no longer required to understand or interpret space" [10]. "What one knows" is the mental set of knowledge to which the perception autonomously refers in order to understand what has been perceived. Is a mechanism that cannot be voluntarily blocked whether or not "the individual is required to understand and interpret"; the individual will however try to do it. Moreover, Eisenman himself acknowledges the imperious demand of the eye and the body to orient itself in architectural space through process of rational "perspectival" ordering. Assertion to share but one point. The word perspectival is superfluous and misleading. In fact, the critics to non-curvilinear, non-folding, and non-topological, non architecture is based on the statement that, until now, the architectural vision has been perspectival. What is not. So the question is displaced. The processes of form generation can be meta-controlled, that is they can be given rules and restraints. Is it enough to guarantee that the process will generate forms susceptible of an interpretation giving meaning to them? A form can be given a sense, a meaning, a value also "a posteriori", as it were a natural object, not an artefact. But this reduces, not enhances the range of its communication capabilities. Two ways are opened to the architect: either they are able to control the output of the form generation - and in this case the form must be, in some way, predictable - or they are not, and in this case they have to accept the randomness of the results [12]. However, this is the new paradigm of design. It changes and, eventually, reduces the responsibility and role of architect in shaping the communication capabilities of his work.

This shaping principle clashes with the opposed one: shapes configured by context forces.

\section{An experiment}

The theory of the influence that contest forces exert on the formal choices of architects sensitive to them seems in the same time, convincing and dubious. Convincing because of its reference to environmental aspect of which an objective presence is asserted. Dubious because this objectivity is subjectively asserted; which seems a patent contradiction.

It seems reasonable to think that much depends on the "strength" of the context, of the presence of highly characterized natural configurations or dynamic phenomena. We decided to perform a simple experiment: the simulation of the deforming activity of "landscape forces" on a project previously designed. The environment is a place at the foot of the mountain 
chain closing the Palermo plain. The mountain behind the lot is the dominant element of the close environment. The hypothesis is that the mountain exerts an attractive force tending to distort "autonomous" volumes, geometrically defined according to only self-referenced configurations. The attraction should fold the volumes towards the mountain. The project chosen as the basis of the transformation experiment was designed in a composition course. It fits well to the experiment, as its shape is geometrically clear, self-centered, and independent from context influences. The project comprises a small airport station with an annexed flight school, a congress center, and a hotel. For simplicity sake the building was simplifies discarding the station and the school. The meeting halls of the congress center acquired the characters of auditoriums. The software tool was 3DS Max $^{\odot}$. Catia $^{\odot}$ was used for some intermediate steps, not reported for brevity sake.

A first phase is a morphing process not immediately bound with the action of forces. The start schema models only the dimensional and relational properties of the single functional spaces. Each of them is represented as a parallelepiped having surface and height as foreseen in the project. The end model is the original project from which some secondary elements have been withdrawn. The procedure is, in a sense, a control of the logic of the original project. In fact it relates the chosen (final) solution with the pure functional requirements of the schema.
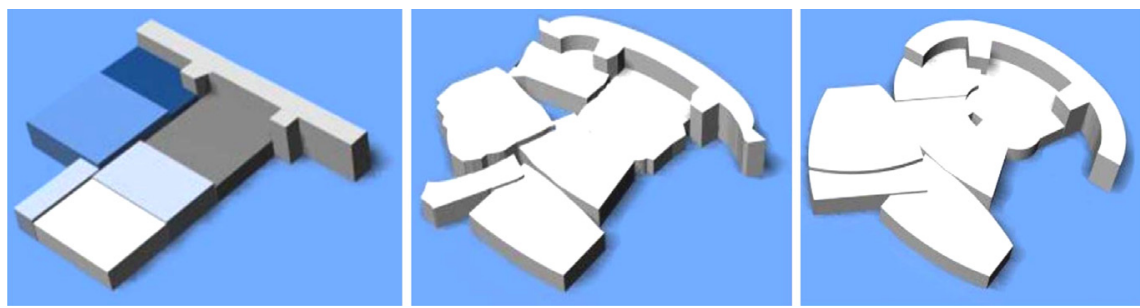

Figure 1: Steps of the morphing procedure. Left: start shape with functional parallelepipeds. Center: intermediate configuration created by the morphing software. Right: final ideal shape from the original project.
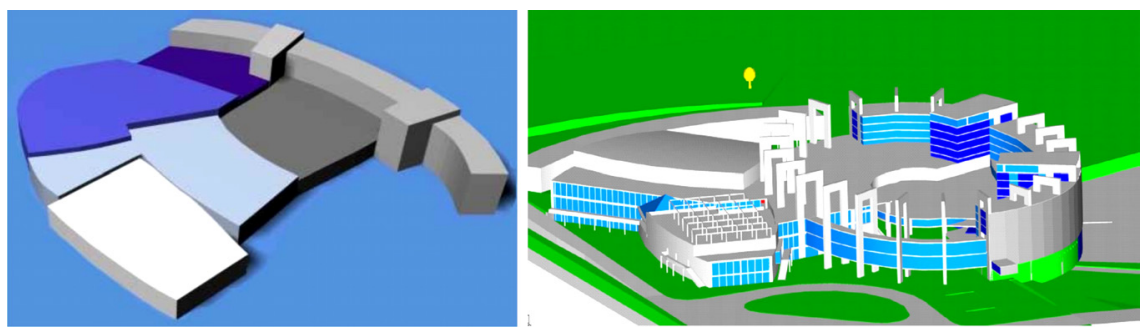

Figure 2: Final shape drawn from the intermediate morphed configuration; and the original project. 


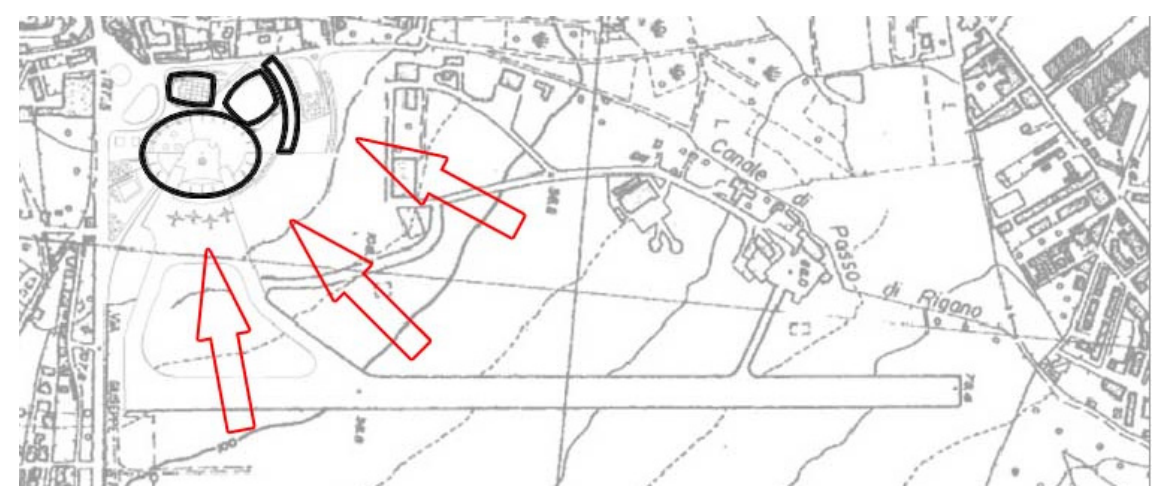

Figure 3: Plan showing the context forces.

The fiftieth configuration out of the hundred generated by the morphing was chosen as a good compromise between pure functional requirement and mainly formal criterion. Small adjustments were hand made.

The following phase brings the context forces. The volume most sensible to the attraction of the dominant mountain is the hotel because of its height and slimness. The most important point of view is the access street. The searched effect has to be well visible from it. The attraction of the mountain was interpreted as a set of forces directed towards it and applied as a thrust to the side of the hotel body opposite to the mountain. This has already been modified (taper) in submission to the context restraints imposed by the airport nearing plans. Besides the contest forces, the sensitivity of the hotel body to them had to be evaluated and simulated. A possible way was to compute the deformations of the hotel body by means of Finite Elements (FE) software, recycling and modifying intensity of forces and/or elasticity of the structure until a visible deformation could be obtained. This way besides being long had an intrinsic contradiction. Elastic deformations are always small enough as not to change the geometric conditions of the static equilibrium; just the contrary of what is needed. 3DS $\operatorname{Max}^{\odot}$ supplies another way: the volume is considered as made of a soft material, whose behavior is conditioned by some parameters as mass, friction, relative density, stiffness damping, air resistance. The acting force is wind. This mechanism of simulation allows much more intense deformations, more deep and irregularly than a FE software could do. In the simulation, some elements are restrained to the soil, representing a certain resistance to the context force, besides the necessary stability. Figure 4 shows the results on both sides of the hotel body.

The results of the experiment are not immediately readable. An analysis of the results does not dissolve the perplexities. The deformations are wide and somewhat irregular. The relationship with the mountain lying behind can be understood only after a careful consideration of some data of the procedure. The shape of the inward flexion of the lee side is a consequence of the restraining effect of the two towers and of the upper chain. On the mountain side an extroflexion can be read as the effect of an attraction, again restrained by the fixed elements of the building. 
Many other aspects of the experiment, as the procedure of communication between $3 \mathrm{DS} \operatorname{Max}^{\odot}$ and $\mathrm{Catia}^{\odot}$ or the use of the FE software present in the latter are not reported because not pertaining to the topic of this paper.
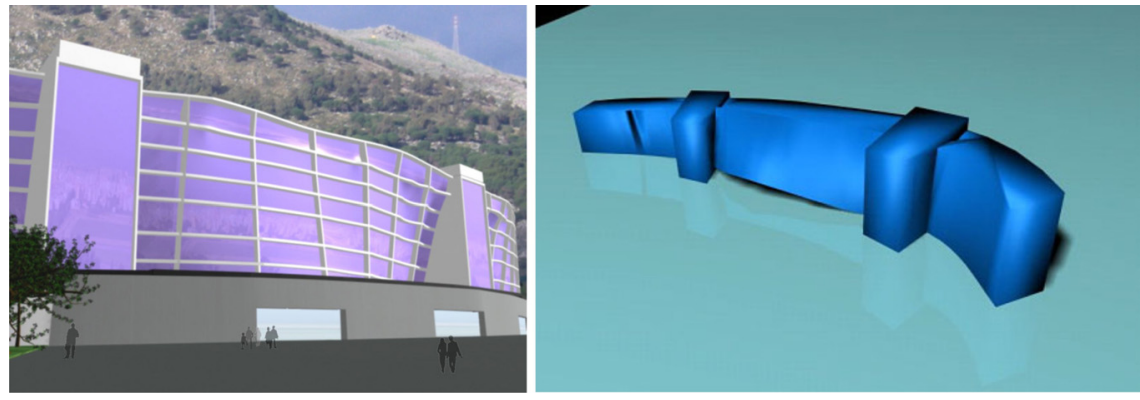

Figure 4: $\quad$ South façade after morphing and north façade after morphing.

\section{Conclusions}

Of course the experiment is very simple and does not pretend to give enough elements to dissipate all the perplexities that arise in such an innovative approach. The morphing procedure is powerful. It allows the reshaping of previous forms as much as desired. Less easy is mastering the results once the object parameters, the restraints, and the force fields are given. We think that the subjectivity of the interpretation of the force fields is substantially confirmed. We do not think that the looseness of correspondence between the hypothesized nature of the context forces and the voluntary model in which they are translated denies reliability to the procedure. It only brings the same looseness in the meaning that the deformed shapes are able to express. However, this is nothing new: subjectivity, i.e. personal individual characterization, has always been the very essence of art and then of architecture. What is wrong is denying this subjectivity.

Something like can be said about the two main assertions of the so called "New Architecture". On one side the pretension that their approach to correspond, interpret, and embody the results of the most advanced science and philosophy [13]. On the other, that only their approach can guarantee such correspondence, interpretation, embodiment. The first statement may hide a substantial weakness and uncertainty, disguised in certitudes, seeking in other disciplines the legitimacy they do not find in their own. We hardly touched on some questionable ways of interpreting mathematical concepts, such as topology or non-Euclidean geometry. Mental schemes having similar abstract conceptual structure can indeed exist in different disciplines [14]. Sometimes, however, the similarity is only apparent, or regards only secondary aspects. What seems hardly convincing is the pretension to appropriate the "aura" of extreme advancement, scientificity, and depth of knowledge, from sometimes only superficial similarities or quite misunderstandings. 
With regards to the second pretension, it is enough to leaf through the magazines of architecture to perceive that a lot of beautiful Euclidean, rectilinear buildings are built the world over.

\section{References}

[1] Lynn G., Architectural curvilinearity. The folder, the pliant and the supple. Architectural Design 3, 1993.

[2] Emmer M., Mathland. Dal mondo piatto alle ipersupefici, Testo e Immagine: Roma, 2003.

[3] MATHWORLD, http://mathworld.wolfram.com

[4] Glymp, J., Evolution of the digital design process (Chapter 8). Architecture in the digital age: design and manufacturing, ed. B. Kolarevic, Spon Press Taylor \&Francis Group: New York and London, pp. 101-120, 2003.

[5] Emmer M., MATHLAND, From Topology to Virtual Architecture. Proc. of Generative Art Conference, ed. Politecnico di Milano: Milano, 2005.

[6] Mitchell W., Roll Over Euclid, How Frank Gehry Designs and Builds. Frank Gehry Architect, ed J. Fiona Ragheb, The Solomon R. Guggenheim Foundation: New York, 2001.

[7] Lenoir T., Alt C., Flow, Process, Fold: intersections in bioinformatics and contemporary Architecture. Science, Metaphor and Architecture, eds. A. Picon and A. Ponte, Princeton University Press: Princeton, 2002.

[8] Kolarevic B., Digital Morphogenesis and Computational Architectures. Constructing the digital space, Proc. of $4^{\text {th }}$ Sigradi Conference, eds. Ripper Kos J., Pessoa Borde A. \& Rodriguez Barros D., Rio de Janeiro, pp. 98-103, 2000.

[9] Franken B., Real as data (Chapter 9). Architecture in the digital age: design and manufacturing, ed. B. Kolarevic, Spon Press Taylor \&Francis Group: New York and London, pp. 121-138, 2003.

[10] Eisenmann P, Visions unfolding architecture in the age of electronic media. Domus 734, pp. 17-24, 1994.

[11] Wöfflin H., Principles of Art History, the Problem of the Development of Style in Later Art, Dover Books on Art. New York: Dover, 1950.

[12] Lynn G., Animate Form, Princeton Architectural Press: New York, 1999.

[13] Imperiale A., Time frozen / Time liberated? New Flatness: Surface Tension in Digital Architecture, 74-8, Birkhäuser - Publishers for Architecture: Basel, 2000.

[14] Emmer M, The Role of Mathematics in Virtual Architecture. Nexus Network Journal 7(2), 2005. 